\title{
On the Implementation of OPF-Based Setpoints for Active Distribution Networks
}

\author{
Michael Z. Liu, Student Member, IEEE, Luis F. Ochoa, Senior Member, IEEE, \\ and Steven H. Low, Fellow, IEEE
}

\begin{abstract}
In the context of active distribution networks, AC Optimal Power Flow (OPF) has shown great potential to calculate setpoints for controllable devices. Although considerable literature exists, temporal aspects that may affect the actual execution of these setpoints are rarely investigated. Due to the diverse operating characteristics of controllable devices (i.e., delays, ramp rates and deadbands), when these setpoints are executed by multiple devices without adequate considerations, the resulting outcome can differ drastically from what is expected; leading to violations of network constraints and excessive control actions. Therefore, this work proposes a series of necessary adaptations within the controllers of existing devices as well as in the OPF formulation to cater for the diversity in operating characteristics, ensuring that calculated setpoints are adequately implemented by controllable devices. This involves the direct control of conventional devices and enforcing a new ramping behavior for inverter-interfaced devices. Furthermore, a linear, mixedinteger formulation is proposed to handle discrete devices and improve scalability in large networks. Co-simulation results (using a UK test network with the objective of maximizing renewable energy production and considering 1s time-step) demonstrate that, by catering for the operating characteristics of controllable devices, the expected outcome from OPF-based setpoints can be achieved.
\end{abstract}

Index Terms-Active distribution network, distributed generation, optimal power flow (OPF), implementation

\section{NOMENCLATURE}

\section{$\underline{\text { Sets }}$}

G DG plants.

$N \quad$ Buses/nodes.

$\Phi \quad$ Phases (electrical).

$S \subset N \quad$ Sources.

$L \quad$ Lines.

$T \quad$ OLTC-fitted transformers.

$\Pi$ Tap positions of OLTCs.

$\underline{\text { Constants/Parameters }}$

$\overline{\boldsymbol{p}} \quad$ Rated capacity of DG plants.

$\boldsymbol{p}^{\text {avail }} \quad$ Renewable resource availability.

M. Z. Liu is with the Department of Electrical and Electronic Engineering, The University of Melbourne, VIC 3010, Australia (e-mail: mliu@ ieee.org).

L. F. Ochoa is with the Department of Electrical and Electronic Engineering, The University of Melbourne, VIC 3010, Australia and the School of Electrical and Electronic Engineering, The University of Manchester, M13 9PL, UK (e-mail: luis_ochoa@ieee.org).

S. H. Low is with the Engineering and Applied Science Division, California Institute of Technology, Pasadena, CA 91125, USA and the Department of Electrical and Electronic Engineering, The University of Melbourne, VIC 3010, Australia (email: slow@ caltech.edu).

\begin{tabular}{|c|c|}
\hline$p^{0}$ & Current active power output of DG plants. \\
\hline$\Delta \bar{p}$ & Power ramp limit of DG plants. \\
\hline$\overline{P F}$ & Power factor capability of DG plants. \\
\hline $\boldsymbol{V}^{+}, \boldsymbol{V}^{-}$ & Upper/lower nodal voltage limits. \\
\hline $\overrightarrow{\boldsymbol{V}}$ & Estimated nodal voltage phasor. \\
\hline$p^{D}, q^{D}$ & Nodal active/reactive power demands. \\
\hline $\boldsymbol{R}, \boldsymbol{X}$ & Physical impedance of lines. \\
\hline $\boldsymbol{R}^{\prime}, \boldsymbol{X}^{\prime}$ & Equivalent impedance of lines due to unbalances. \\
\hline$I^{+}$ & Ampacity of lines. \\
\hline $\boldsymbol{\rho}, \bar{\Delta}, \Lambda$ & $\begin{array}{l}\text { Approximation constants to model the thermal } \\
\text { capacity of lines. }\end{array}$ \\
\hline $\boldsymbol{\tau}$ & Tap ratio of a given tap position. \\
\hline$\tau^{+}, \tau^{-}$ & Upper/lower limits of feasible tap ratios. \\
\hline$\pi^{0}$ & Current tap position of OLCT-fitted transformers. \\
\hline$M$ & Big $\mathrm{M}$ in disjunctive constraints. \\
\hline $\boldsymbol{c}$ & Scaling factors in the objective function. \\
\hline
\end{tabular}

$\underline{\text { Variables }}$

$p, q \quad$ Active/reactive power output of DG plants.

$v \quad$ Squared, line-to-neutral voltage magnitudes.

$p^{S}, q^{S} \quad$ Active/reactive power exchange with sources.

$P, Q \quad$ Active/reactive power flow into the start/end of lines/transformers.

$P^{+}, P^{-}$

$Q^{+}, Q^{-}$,

$\Delta^{P}, \Delta^{Q}, \lambda$

$\pi^{*} \quad$ Tap position of OLTC-fitted transformers.

$k \quad$ Binary state (active/inactive) of tap positions.

$\kappa, H \quad$ Auxiliary terms introduced (for compactness).

$u$

Control effort/volume of control actions.

\section{INTRODUCTION}

$\mathrm{T}$ HE uptake of renewable Distributed Generation (DG) in distribution networks is creating significant challenges for Distribution Network Operators (DNOs) to cope with steadystate network issues such as voltage rise and congestion. An alternative to the otherwise required network reinforcements is the use of active network management; a concept that has already been demonstrated to be viable and cost-effective in early trials [1]. In this context, operators of these active distribution networks will need to determine, on a minute scale, appropriate setpoints for the controllable devices to achieve the desired objective without violating network constraints.

In recent literature, many works (e.g., [2-5]) have shown the potential of using AC Optimal Power Flow (OPF), a widely-known mathematical optimization problem, to calculate 
optimal setpoints for controllable devices in constrained distribution networks. There are also efforts in tailoring the OPF for specific scenarios, such as dealing with the uncertainty in renewable resources [6-8]. However, since these studies typically focus on the calculation of setpoints (and use minutely/hourly time-steps), temporal aspects in the sub-minute scale that may affect the outcomes when these setpoints are implemented in actual networks are rarely investigated.

As the execution of setpoints do not happen instantaneously, the diversity in operating characteristics (i.e., delays, ramp rates and deadbands) of different controllable devices may lead to outcomes that differ drastically from what is expected by the OPF; potentially leading to network constraint violations and excessive control actions. This aspect is of particularly relevance in the context of voltage management schemes given that there are often multiple avenues to address voltage issues (e.g., [9-11]), such as voltage regulation assets-onload tap changers (OLTCs) and capacitor banks - as well as the reactive power capability of inverter-interfaced DG plants.

For instance, the delay of conventional OLTC controllers (typically over a minute) may cause temporal coordination issues with faster acting devices (e.g., inverter-interfaced DG plants), resulting in temporary voltages spikes before all the requested setpoints are reached. In fact, the coordination issue between wind farms and OLTCs has already been identified in the UK-based Flexible Plug and Play (FPP) project [12]. As a result, the maximum ramp rate of wind farms was limited in the FPP project to cater for the delay of conventional OLTC controllers. At the same time, following this principle, if the response of DG plants is restricted (e.g., due to a ramp rate limit), this may lead to similar coordination issues with other devices that have much faster responses to new setpoints. Lastly, since an OLTC controller is unable to strictly follow a voltage target calculated by the OPF due to its deadbands, they become less compatible with OPF-based schemes-an important aspect that, to the best of the authors' knowledge, is yet to be investigated in the existing literature.

Since the adoption of OPF-based schemes are increasingly being considered by DNOs [13, 14], ensuring that the calculated setpoints are adequately implemented by controllable devices is crucial. Consequently, their delays, ramp rates and deadbands must be accounted for. Furthermore, the above also highlights that, to fully investigate any potential issues, a cosimulation environment with high time resolution (i.e., using a sub-minute time-step [15]) is necessary to distinguish the separation between the calculation of setpoints and the implementation of setpoints.

To this end, techniques to perform OPF in the seconds scale have been proposed in [16, 17]. However, the general focus was on solution algorithms and their speed; challenges associated with the implementation of setpoints were not addressed. The need for a co-simulation environment was highlighted in [18], where, using a 5-second time-step, the authors showed that delays and deadbands of controllable devices can result in voltage issues when setpoints (calculated by OPF) are implemented. To mitigate such issues, a small penalty factor was used in the objective function to find setpoints that steer

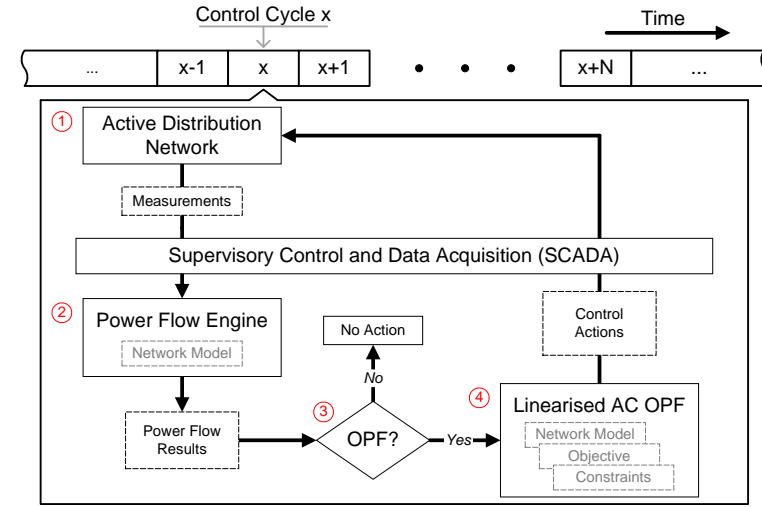

Fig. 1. Control architecture

voltages away from the statutory limits; effectively creating a margin to accommodate any voltage fluctuations. However, this solution is mostly effective when reverse power flows (and, thus, voltage rise) are not significant. With large reverse power flows, voltages are closer to the limit and, thus, this solution is not able to create further margin.

A key contribution of this work is highlighting 1) the need for practical considerations when OPF-based setpoints are to be implemented by the DNO; and, 2) the limitations of conventional device controllers in the context of active distribution networks. To address these practical challenges, this work also proposes a series of necessary adaptations within the controllers of existing devices and the OPF formulation. For conventional voltage regulation devices (e.g., OLTCs), direct control is adopted to address the limitations of autonomous controllers (i.e., prolonged delays and large deadbands). For inverter-interfaced DG plants, a new ramping strategy is proposed to achieve better temporal coordination with conventional devices. As for the OPF, a linear, mixed-integer formulation is proposed to cater for discrete devices and improve scalability for large networks. The operating limits of controllable devices (e.g., the ramp rate) are also incorporated as constraints to ensure the calculated setpoints are feasible within the considered timeframe.

\section{IMPLEMENTATION ISSUES OF OPF-BASED SETPOINTS AND PROPOSED ADAPTATIONS}

Fig. 1 shows the control architecture for an active distribution network using minute-scale control cycles. There are four key steps in each control cycle, which are indicated by (1) to (4) in Fig. 1. The first step, i.e., (1), involves collecting the necessary measurements to assess constraint violations (e.g., demands, output and setpoints of DG plants, and setpoints of conventional devices) through the SCADA infrastructure. These measurements are used in combination with the network model to check for voltage and/or congestion issues by running a three-phase power flow, this is indicated by (2). The power flow results are then used to determine whether an OPF calculation is needed, as indicated by (3). If so, a three-phase AC OPF (presented in Section III) is solved to determine the most adequate setpoints, as indicated by (4). Once calculated, the new setpoints are dispatched to the controllable devices and maintained throughout the corresponding control cycle. 
Since the implementation of setpoints is handled by the devices, their diverse delays, ramp rates and deadbands may affect how these setpoints are achieved, and thus, the overall performance. The following subsections first discuss the operating characteristics of the controllable devices and the potential issues. Based on this, the proposed adaptations to cater for delays, ramp rates and deadbands are described.

\section{A. Operating Characteristics}

In general, conventional voltage regulation devices are designed to operate autonomously. For instance, an OLTC is designed to regulate the voltage of a specific node/bus by changing its taps. To prevent excessive tap changes due to temporary voltage fluctuations, tap changes are only triggered if the voltage deviates outside a deadband (typically 1-2\% from the voltage target) for a period longer than the delay (typically 1-2 minutes). After this initial delay, the physical movement of taps to restore the voltage (i.e., the ramp rate, although discrete in nature) is much faster, only a few seconds per tap. Voltage-based controllers for capacitor banks also exhibit similar characteristics.

In contrast, these operating characteristics are drastically different for modern DG plants. Upon receiving the requested setpoints, the initial delay is often negligible (e.g., mere seconds). However, there are often restrictions on their ramp rates to prevent extreme fluctuations in active power outputs. Consequently, the requested setpoint may require minutes to reach. Though less common, similar restrictions on ramp rates can also be applied to the reactive power/power factor. Lastly, due to the continuous nature, deadbands (like those found in conventional devices) are typically not needed.

\section{B. Potential Issues}

The first issue relates to temporal coordination among controllable devices; particularly in terms of their delays and ramp rates. To demonstrate this, a timing diagram of the critical events during a control cycle (not necessarily to scale) are shown for two scenarios: uncoordinated (Fig. 2.a) and coordinated (Fig. 2.b). The start and end of a control cycle are denoted by $\mathbf{0}$ and $\boldsymbol{T}_{\mathrm{CC}}$, respectively. The computation time (to run the power flow and solve the OPF) and communication delay are represented by $\Delta T_{\mathrm{OPF}}$ and $\Delta T_{\mathrm{COM}}$, respectively. The remaining $\Delta$ terms represent the times required for each controllable device to fully reach the requested setpoints, henceforth referred to as their execution times. Here, the execution times of DG plants $\left(\Delta T_{D G}^{P}\right.$ for active power and $\Delta T_{D G}^{P F}$ for power factor) and OLTCs ( $\Delta T_{\text {OLTC }}$ for tap changes) are shown. Within each execution time, the delay component is highlighted by the shaded region; it refers to the period before a physical response is initiated after receiving the setpoints. Similarly, the duration of the physical response to reach the new setpoint, considering the applicable ramp rate, is highlighted by the solid region.

The uncoordinated scenario (Fig. 2.a) happens when the execution times of different devices are significantly out of alignment. Despite receiving the setpoint at the same time (i.e., after $\Delta T_{\mathrm{OPF}}+\Delta T_{\mathrm{COM}}$ ), there is a noticeable delay before all calculated setpoints are reached (as indicated by $T^{*}$ ). Con-

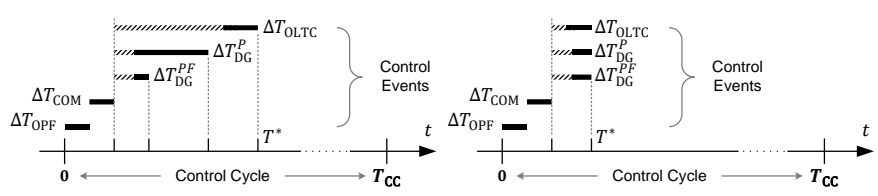

a. Uncoordinated

b. Coordinated

Fig. 2. Timing of critical control events in a control cycle

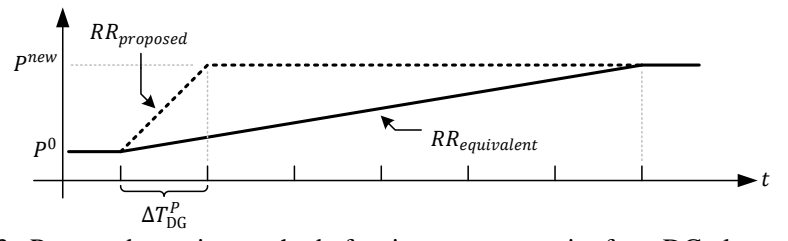

Fig. 3. Proposed ramping method of active power setpoint for a DG plant

sequently, unexpected outcomes differ to that expected by the OPF may arise during this transition period (before $T^{*}$ ). In contrast, Fig. 2.b illustrates a more desirable scenario where all execution times are aligned (i.e., coordinated) to mitigate adverse impacts during the transition period.

In addition to causing coordination issues, significantly long execution times are also undesirable when control cycles are used given that the requested setpoint for a control cycle may not be achieved. For instance, if the delay of an OLTC is longer than the control cycle, the requested actions will not happen within that control cycle.

The last issue arises from the deadband of conventional devices. While a decision-making algorithm can calculate the optimal voltage target, due to the presence of a deadband, these devices are unable to strictly follow the precise voltage target calculated by the OPF. Instead, the actual voltage measured at the regulated node/bus may drift around the requested target (albeit still within the deadband). Consequently, this voltage drift may lead to unintended behaviors at downstream sections of the network. The severity is further exacerbated if there are multiple devices with deadbands along the path.

\section{Adaptations to Cater for Delays, Ramps and Deadbands}

For OLTCs, the direct control method is proposed to reduce the delays and achieve more swift responses. This approach bypasses the existing voltage target-based OLTC controller by commanding the require tap position directly. As such, the tap positions are also considered as variables in the OPF. Since the problematic delays (associated with existing OLTC controllers) are eliminated, both OLTCs and DG plants can respond immediately after receiving the setpoints.

Due to ramp rate restrictions on DG plants, there is an inherent limit on how quickly the setpoints can be achieved, and thus, resulting in undesirably longer execution times (despite having negligible delay). In terms of active power, since the ramp rate is often specified in MW/min, how it is enforced in the sub-minute scale can be exploited to achieve the desirable execution time. Fig. 3 illustrates two ways of achieving the same ramp rate on the minute scale but very different execution times on the sub-minute scale: a linear and constant ramp (solid line) and a faster initial ramp (dashed line). In this work, the latter approach is adopted where an initial fast ramp is tuned to achieve the desirable execution time while respecting the imposed ramp rate in $\mathrm{MW} / \mathrm{min}$. As such, the OPF must 
also consider both the connection agreement ramp rate and the full ramping capability as constraints to ensure that the requested setpoint is technically feasible. If needed, the same approach can also be applied to the reactive power.

Finally, the proposed direct control method (of OLTCs) also eliminates the associated deadbands, as it allows the precise tap positions (calculated by the OPF) to be implemented in practice. Therefore, no additional changes are required.

\section{Remarks on the Proposed Adaptations}

Although the direct control of OLTCs-i.e., setting the tap position directly instead of commanding a voltage target-is an uncommon practice in existing distribution networks, its technical feasibility has already been demonstrated (e.g., in the Customer Load Active System Services project [19]). Nonetheless, this may entail infrastructure upgrades to incompatible OLTC controllers. Furthermore, while the proposed scheme commands the precise tap position, existing voltage-based control mechanism can still be retained as a fail-safe mode to cater for unexpected events (e.g., extreme voltages or loss of communication). As for DG plants, the ability to receive external signals has also been demonstrated (e.g., in [1]). Furthermore, the technical feasibility of the proposed ramping behavior (i.e., in Fig. 3) is ensured through both constraint (22) and the fact that inverter-interfaced devices typically can fully ramp up/down in the seconds scale [20].

\section{THREE-PHASE AC OPF}

The three-phase AC OPF is formulated as a mixed-integer linear program (MILP). It is adapted from the authors' previous works [21, 22], which uses the branch flow model (BFM) [23] for the power flow equations. Specifically, the threephase formulation presented in [22] is extended with additional constraints to cater for the operating characteristics of controllable devices. For completeness, the linearization process and the usage of estimated values is outlined in the Appendix. Integer variables are introduced to cater for the discrete nature of OLTCs. Numerical constants are highlighted in bold (to differentiate from optimization variables) and per unit (p.u.) values are used where applicable. Complex vectors/phasors are denoted by the accent $\overrightarrow{ }$. .

The objective of maximizing renewable energy production while minimizing the control actions is given by (1), where the active power output of a DG plant $g$ in phase $\phi$ is denoted by $p_{g, \phi}$. The control effort (i.e., volume of control actions) associated with OLTCs and DG plants are given by $u_{t}$ and $u_{g}$, respectively. The scaling factors, $\boldsymbol{c}_{\mathbf{1}}, \boldsymbol{c}_{\mathbf{2}}$ and $\boldsymbol{c}_{\mathbf{3}}$, are determined based on priorities from a technical perspective to reflect the primary objective of maximizing energy production. Hence, they are selected such that the maximum value of $\boldsymbol{c}_{\mathbf{2}} \sum u_{t}+\boldsymbol{c}_{\mathbf{3}} \sum u_{g}$ will have minimal impact on $\boldsymbol{c}_{\mathbf{1}} \sum \sum p_{g, \phi}$, while still within the tolerances of the solver to penalize an increase in control effort for negligible benefits. This can be done by assigning values to the scaling factors that normalize each summation (considering their ceilings) and are of different order of magnitudes (based on their priorities).

$$
\max \left(\boldsymbol{c}_{\mathbf{1}} \sum_{g \in G} \sum_{\phi \in \Phi} p_{g, \phi}\right)-\left(\boldsymbol{c}_{\mathbf{2}} \sum_{t \in T} u_{t}+\boldsymbol{c}_{\mathbf{3}} \sum_{g \in G} u_{g}\right)
$$

\section{A. Distribution Network Modelling}

Voltage constraints for the set of nodes $N$ (indexed by $n$ ) are given in (2). The squared, line-to-neutral voltage magnitudes (denoted by $v_{n, \phi}$ ) are considered as the optimization variable. The upper and lower limits are denoted by $\boldsymbol{V}_{\boldsymbol{n}}^{+}$and $\boldsymbol{V}_{\boldsymbol{n}}^{-}$, respectively. The phases are represented in $\Phi=\{\mathrm{A}, \mathrm{B}, \mathrm{C}\}$.

$$
\left(\boldsymbol{V}_{\boldsymbol{n}}^{-}\right)^{2} \leq v_{n, \phi} \leq\left(\boldsymbol{V}_{n}^{+}\right)^{2}, \forall n \in N, \phi \in \Phi
$$

The power flows into the set of lines $L$ (indexed by $l$ ) from the start node and end node are given by the function in (3)(5). Here, $l_{x} \in N$ and $l_{y} \in N$ denote the start node and end node of line $l$, respectively. The convention of $P$ for active power and $Q$ for reactive power is used. Repeated expressions are represented by auxiliary terms " $\kappa$ " for compactness. Estimate values of the variables (denoted by accent $\sim$ ) are used to linearize the quadratic terms in the BFM, as proposed in [21]. For instance, the estimated terms $\widetilde{\boldsymbol{\kappa}}_{\mathbf{1}}$ and $\widetilde{\boldsymbol{\kappa}}_{\mathbf{2}}$ can be obtained from the corresponding variables $\kappa_{1}$ and $\kappa_{2}$, respectively, by substituting $\left(\widetilde{\boldsymbol{P}}_{\boldsymbol{l}_{\boldsymbol{x}}, \boldsymbol{\psi}}, \widetilde{\boldsymbol{Q}}_{\boldsymbol{l}_{\boldsymbol{x}}, \boldsymbol{\psi}}\right)$ for $\left(P_{l_{x}, \psi}, Q_{l_{x}, \psi}\right)$.

$$
\begin{aligned}
& v_{l_{x}, \phi}-v_{l_{y}, \phi}=2 \kappa_{1}-\left(\kappa_{1} \widetilde{\boldsymbol{\kappa}}_{\mathbf{1}}+\kappa_{2} \widetilde{\boldsymbol{\kappa}}_{2}\right) / \widetilde{\boldsymbol{v}}_{l_{x}, \boldsymbol{\phi}} \\
& P_{l_{y}, \phi}=-\left(P_{l_{x}, \phi}-\left(P_{l_{x}, \phi} \widetilde{\boldsymbol{\kappa}}_{3}-Q_{l_{x}, \phi} \widetilde{\boldsymbol{\kappa}}_{\mathbf{4}}\right) / \widetilde{\boldsymbol{v}}_{l_{x}, \boldsymbol{\phi}}\right) \\
& Q_{l_{y}, \phi}=-\left(Q_{l_{x}, \phi}-\left(P_{l_{x}, \phi} \widetilde{\boldsymbol{\kappa}}_{\mathbf{4}}+Q_{l_{x}, \phi} \widetilde{\boldsymbol{\kappa}}_{3}\right) / \widetilde{\boldsymbol{v}}_{\boldsymbol{l}_{x}, \boldsymbol{\phi}}\right) \\
& \forall l \in L, \forall \phi \in \Phi \\
& \kappa_{1}=\sum_{\psi \in \Phi}\left(\boldsymbol{R}_{l, \phi, \psi}^{\prime} P_{l_{x}, \psi}+\boldsymbol{X}_{l, \phi, \psi}^{\prime} Q_{l_{x}, \psi}\right) \\
& \kappa_{2}=\sum_{\psi \in \Phi}\left(\boldsymbol{X}_{\boldsymbol{l}, \boldsymbol{\phi}, \boldsymbol{\psi}}^{\prime} P_{l_{x}, \psi}-\boldsymbol{R}_{\boldsymbol{l}, \boldsymbol{\phi}, \boldsymbol{\psi}}^{\prime} Q_{l_{x}, \psi}\right) \\
& \widetilde{\boldsymbol{\kappa}}_{\mathbf{3}}=\sum_{\psi \in \Phi}\left(\boldsymbol{R}_{\boldsymbol{l}, \boldsymbol{\phi}, \boldsymbol{\psi}}^{\prime} \widetilde{\boldsymbol{P}}_{\boldsymbol{l}_{\boldsymbol{x}}, \boldsymbol{\psi}}+\boldsymbol{X}_{l, \phi, \psi}^{\prime} \widetilde{\boldsymbol{Q}}_{\boldsymbol{l}_{x}, \psi}\right) \\
& \widetilde{\boldsymbol{\kappa}}_{\boldsymbol{4}}=\sum_{\psi \in \Phi}\left(\boldsymbol{X}_{\boldsymbol{l}, \boldsymbol{\phi}, \boldsymbol{\psi}}^{\prime} \widetilde{\boldsymbol{P}}_{\boldsymbol{l}_{x}, \boldsymbol{\psi}}-\boldsymbol{R}_{\boldsymbol{l}, \boldsymbol{\phi}, \boldsymbol{\psi}}^{\prime} \widetilde{\boldsymbol{Q}}_{\boldsymbol{l}_{\boldsymbol{x}}, \boldsymbol{\psi}}\right)
\end{aligned}
$$

The equivalent impedance $\boldsymbol{Z}_{\boldsymbol{l}, \boldsymbol{\phi}, \boldsymbol{\psi}}^{\prime}=\boldsymbol{R}_{\boldsymbol{l}, \boldsymbol{\phi}, \boldsymbol{\psi}}^{\prime}+j \boldsymbol{X}_{\boldsymbol{l}, \boldsymbol{\phi}, \boldsymbol{\psi}}^{\prime}$ can be approximated per (6) and (7) using the physical impedance $\boldsymbol{Z}_{\boldsymbol{l}, \boldsymbol{\phi}, \boldsymbol{\psi}}=\boldsymbol{R}_{\boldsymbol{l}, \boldsymbol{\phi}, \boldsymbol{\psi}}+j \boldsymbol{X}_{\boldsymbol{l}, \boldsymbol{\phi}, \boldsymbol{\psi}}$ and estimated voltage magnitudes and angles. These values come from the power flow results as shown in Fig. 1.

$$
\begin{aligned}
& \boldsymbol{R}_{l, \phi, \psi}^{\prime}=\left[\boldsymbol{R}_{\boldsymbol{l}, \boldsymbol{\phi}, \boldsymbol{\psi}} \cos \left(\widetilde{\boldsymbol{\kappa}}_{\mathbf{5}}\right)-\boldsymbol{X}_{\boldsymbol{l}, \boldsymbol{\phi}, \boldsymbol{\psi}} \sin \left(\widetilde{\boldsymbol{\kappa}}_{\mathbf{5}}\right)\right] \widetilde{\boldsymbol{V}}_{\boldsymbol{l}_{\boldsymbol{x}}, \boldsymbol{\phi}} / \widetilde{\boldsymbol{V}}_{\boldsymbol{l}_{\boldsymbol{x}}, \boldsymbol{\psi}} \\
& \boldsymbol{X}_{l, \phi, \psi}^{\prime}=\left[\boldsymbol{R}_{\boldsymbol{l}, \boldsymbol{\phi}, \boldsymbol{\psi}} \sin \left(\widetilde{\boldsymbol{\kappa}}_{\mathbf{5}}\right)+\boldsymbol{X}_{\boldsymbol{l}, \boldsymbol{\phi}, \boldsymbol{\psi}} \cos \left(\widetilde{\boldsymbol{\kappa}}_{\mathbf{5}}\right)\right] \widetilde{\boldsymbol{V}}_{\boldsymbol{l}_{\boldsymbol{x}}, \boldsymbol{\phi}} / \widetilde{\boldsymbol{V}}_{\boldsymbol{l}_{\boldsymbol{x}}, \boldsymbol{\psi}} \\
& \forall l \in L, \forall \phi \in \Phi, \forall \psi \in \Phi \\
& \widetilde{\boldsymbol{\kappa}}_{\mathbf{5}}=\angle \overrightarrow{\boldsymbol{V}}_{\boldsymbol{l}_{\boldsymbol{x}}, \boldsymbol{\psi}}-\angle \overrightarrow{\boldsymbol{V}}_{\boldsymbol{l}_{\boldsymbol{x}}, \boldsymbol{\phi}}
\end{aligned}
$$

The thermal limit of a line for a given ampacity $\boldsymbol{I}_{l}^{+}$, i.e., $P_{l_{x}, \phi}^{2}+Q_{l_{x}, \phi}^{2} \leq v_{l_{x}, \phi}\left(I_{l}^{+}\right)^{2}$, is approximated using the piecewise linearization technique in [21], as shown in (8)-(14).

$$
\begin{aligned}
& \sum_{\lambda} \boldsymbol{\rho}_{l, \boldsymbol{\phi}, \lambda}\left(\Delta_{l_{x}, \phi, \lambda}^{P}+\Delta_{l_{x}, \phi, \lambda}^{Q}\right) \leq v_{l_{x}, \phi}\left(I_{l}^{+}\right)^{2} \\
& P_{l_{x}, \phi}=P_{l_{x}, \phi}^{+}-P_{l_{x}, \phi}^{-} \quad Q_{l_{x}, \phi}=Q_{l_{x}, \phi}^{+}-Q_{l_{x}, \phi}^{-} \\
& P_{l_{x}, \phi}^{+}+P_{l_{x}, \phi}^{-}=\sum_{\lambda} \Delta_{l_{x}, \phi, \lambda}^{P} \quad Q_{l_{x}, \phi}^{+}-Q_{l_{x}, \phi}^{-}=\sum_{\lambda} \Delta_{l_{x}, \phi, \lambda}^{Q} \\
& 0 \leq \Delta_{l_{x}, \phi, \lambda}^{P} \leq \bar{\Delta}_{l, \phi} \quad 0 \leq \Delta_{l_{x}, \phi, \lambda}^{Q} \leq \bar{\Delta}_{l, \phi} \\
& P_{l_{x}, \phi}^{+}, P_{l_{x}, \phi}^{-}, Q_{l_{x}, \phi}^{+}, Q_{l_{x}, \phi}^{-} \geq 0 \\
& \boldsymbol{\rho}_{l, \phi, \lambda}=(2 \lambda-1) \bar{\Delta}_{l, \phi} \\
& \bar{\Delta}_{l, \phi}=V_{n, \phi}^{+} I_{l}^{+} / \Lambda \\
& \forall l \in L, \forall \phi \in \Phi, \forall \lambda \in\{1,2, \ldots, \Lambda\}
\end{aligned}
$$


A three-phase, OLTC-fitted, delta-wye-connected transformer $t$ (in the set $T$ ) is modelled by a lossless transformer connected in series with three single-phase lines at the end of each secondary winding, as shown in Fig. 4. The OLTC is assumed to be installed on the primary winding of the transformer. Using the same nomenclature as distribution lines, the start node and end node voltages for phase $\phi$ are $v_{t_{x}, \phi}$ and $v_{t_{y}, \phi}$, respectively. Similarly, the power flow terms are $P_{t_{x}, \phi}$, $P_{t_{y}, \phi}, Q_{t_{x}, \phi}$ and $Q_{t_{y}, \phi}$. Since line segments are needed to model the losses, additional terms for the lossless transformer (ones with superscripts ${ }^{p r i}$ and ${ }^{s e c}$ ) are introduced for better clarity in (15)-(17). Their relationships with respect to the physical transformer are illustrated in Fig. 4 for phase $A$.

The power relationships for the lossless transformer are derived from [24], as shown in matrix form in (15) and (16).

$$
\begin{aligned}
& {\left[\begin{array}{l}
P_{t, A}^{p r i} \\
P_{t, B}^{p r i} \\
P_{t, C}^{\text {pri }}
\end{array}\right]=\frac{1}{2}\left[\begin{array}{lll}
1 & 1 & 0 \\
0 & 1 & 1 \\
1 & 0 & 1
\end{array}\right]\left[\begin{array}{l}
P_{t, A}^{s e c} \\
P_{t, B}^{s e c} \\
P_{t, C}^{s e c}
\end{array}\right]+\frac{1}{2 \sqrt{3}}\left[\begin{array}{ccc}
1 & -1 & 0 \\
0 & 1 & -1 \\
-1 & 0 & 1
\end{array}\right]\left[\begin{array}{l}
Q_{t, A}^{s e c} \\
Q_{t, B}^{s e c} \\
Q_{t, C}^{s e c}
\end{array}\right]} \\
& {\left[\begin{array}{l}
Q_{t, A}^{\text {sec }} \\
Q_{t, B}^{\text {pri }} \\
Q_{t, C}^{\text {pri }}
\end{array}\right]=\frac{1}{2}\left[\begin{array}{lll}
1 & 1 & 0 \\
0 & 1 & 1 \\
1 & 0 & 1
\end{array}\right]\left[\begin{array}{l}
Q_{t, A}^{s e c} \\
Q_{t, B}^{s e c} \\
Q_{t, C}^{s e c}
\end{array}\right]-\frac{1}{2 \sqrt{3}}\left[\begin{array}{ccc}
1 & -1 & 0 \\
0 & 1 & -1 \\
-1 & 0 & 1
\end{array}\right]\left[\begin{array}{l}
P_{t, A}^{s e c} \\
P_{t, B}^{s e c} \\
P_{t, C}^{s e c}
\end{array}\right]}
\end{aligned}
$$

$\forall t \in T$

Voltage regulation constraints for the lossless transformer are shown in (17) where $\boldsymbol{\tau}_{\boldsymbol{t}}^{-}$and $\boldsymbol{\tau}_{\boldsymbol{t}}^{+}$corresponds to the minimum and maximum tap ratio, respectively. The auxiliary term $\mathrm{H}_{t}$ is introduced for compactness and the superscript ${ }^{t r}$ represents the matrix transpose. In a perfectly balanced system, the second column vector on the right side of $\mathrm{H}_{t}$ equals $\left[\begin{array}{lll}0 & 0 & 0\end{array}\right]^{t r}$. On the other hand, this is no longer true for unbalanced networks. Similar to lines, power flow results (i.e., voltages $\overrightarrow{\boldsymbol{V}}_{\boldsymbol{t}, \boldsymbol{A}}^{\boldsymbol{p r i}}, \overrightarrow{\boldsymbol{V}}_{\boldsymbol{t}, \boldsymbol{B}}^{p r i}$ and $\overrightarrow{\boldsymbol{V}}_{\boldsymbol{t}, \boldsymbol{C}}^{\text {pri }}$ ) can be used to improve the accuracy as proposed in [22].

$$
\begin{aligned}
& 3\left(\boldsymbol{\tau}_{t}^{-}\right)^{2}\left[\begin{array}{lll}
v_{t, A}^{s e c} & v_{t, B}^{s e c} & v_{t, C}^{s e c}
\end{array}\right]^{t r} \leq \mathrm{H}_{t} \leq \\
& 3\left(\boldsymbol{\tau}_{t}^{+}\right)^{2}\left[\begin{array}{lll}
v_{t, A}^{s e c} & v_{t, B}^{s e c} & v_{t, C}^{s e c}
\end{array}\right]^{t r}, \forall t \in T \\
& \mathrm{H}_{t}=\left[\begin{array}{ccc}
2 & -1 & 2 \\
2 & 2 & -1 \\
-1 & 2 & 2
\end{array}\right]\left[\begin{array}{c}
v_{t, \boldsymbol{A}}^{p r i} \\
v_{t, B}^{\text {pri }} \\
v_{t, C}^{\text {pri }}
\end{array}\right]-\left[\begin{array}{c}
\left|\overrightarrow{\boldsymbol{V}}_{\boldsymbol{t}, \boldsymbol{A}}^{\text {pri }}+\overrightarrow{\boldsymbol{V}}_{\boldsymbol{t}, \boldsymbol{C}}^{\text {pri }}\right|^{2}-\left|\overrightarrow{\boldsymbol{V}}_{\boldsymbol{t}, \boldsymbol{B}}^{\text {pri }}\right|^{2} \\
\left|\overrightarrow{\boldsymbol{V}}_{\boldsymbol{t}, \boldsymbol{A}}^{\text {pri }}+\overrightarrow{\boldsymbol{V}}_{\boldsymbol{t}, \boldsymbol{B}}^{\text {pri }}\right|-\left|\overrightarrow{\boldsymbol{V}}_{\boldsymbol{t}, \boldsymbol{C}}^{\text {pri }}\right|^{2} \\
\left|\overrightarrow{\boldsymbol{V}}_{\boldsymbol{t}, \boldsymbol{B}}^{\text {pri }}+\overrightarrow{\boldsymbol{V}}_{\boldsymbol{t}, \boldsymbol{C}}^{\text {pri }}\right|-\left|\overrightarrow{\boldsymbol{V}}_{\boldsymbol{t}, \boldsymbol{A}}^{\text {pri }}\right|^{2}
\end{array}\right]
\end{aligned}
$$

The losses and thermal limit of the transformer are modelled by the single-phase line segments (as shown in Fig. 4) with impedance values matching the transformer's [25]. The corresponding power flow equations can be extracted from (3)-(14) by only considering a single phase in $\Phi$ and are omitted for compactness.

The interconnections (e.g., upstream network) are represented by the set $S \subset N$, indexed by $s$. These interconnections can import or export both active and reactive power as required, i.e. $p_{s, \phi}^{S} \in \mathbb{R}$ and $q_{s, \phi}^{S} \in \mathbb{R}$. Additionally, one of these buses is selected as the slack bus.

The overall power balance at each bus in the network is described by Kirchhoff's Current Law, as shown in (18) and (19). Here, $\boldsymbol{p}_{n, \boldsymbol{\phi}}^{\boldsymbol{D}}$ and $\boldsymbol{p}_{n, \boldsymbol{\phi}}^{\boldsymbol{D}}$ are the active and reactive demand

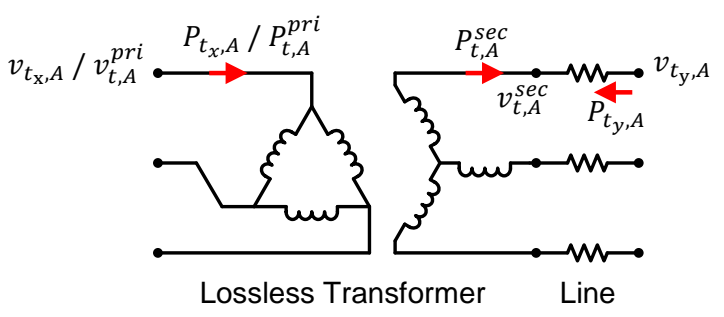

Fig. 4. Delta-wye-connected transformer

at node $n$, respectively. The term $g_{x} \in N$ represents the nodal connection of DG plant $g$.

$$
\begin{gathered}
\sum_{\left(g \in G \mid g_{x}=n\right)} p_{g, \phi}+\sum_{(s \in S \mid s=n)} p_{s, \phi}^{S}=\boldsymbol{p}_{n, \phi}^{D}+ \\
\sum_{\left(l \in L \mid l_{x}=n\right)} P_{l_{x}, \phi}+\sum_{\left(l \in L \mid l_{y}=n\right)} P_{l_{y}, \phi}+ \\
\sum_{\left(t \in T \mid t_{x}=n\right)} P_{t_{x}, \phi}+\sum_{\left(t \in T \mid t_{y}=n\right)} P_{t_{y}, \phi} \\
\sum_{\left(g \in G \mid g_{x}=n\right)} q_{g, \phi}+\sum_{(s \in S \mid s=n)} q_{s, \phi}^{S}=\boldsymbol{q}_{n, \phi}^{D}+ \\
\sum_{\left(l \in L \mid l_{x}=n\right)} Q_{l_{x}, \phi}+\sum_{\left(l \in L \mid l_{y}=n\right)} Q_{l_{y}, \phi}+ \\
\sum_{\left(t \in T \mid t_{x}=n\right)} Q_{t_{x}, \phi}+\sum_{\left(t \in T \mid t_{y}=n\right)} Q_{t_{y}, \phi} \\
\forall n \in N, \phi \in \Phi
\end{gathered}
$$

\section{B. Controllable Devices}

Both the active $\left(p_{g, \phi}\right)$ and reactive $\left(q_{g, \phi}\right)$ power of a DG plant $g$ are considered as controllable. The active power is limited by its rated capacity $\left(\overline{\boldsymbol{p}}_{\boldsymbol{g}, \boldsymbol{\phi}}\right)$ and the resource availability $\left(\boldsymbol{p}_{\boldsymbol{g}, \boldsymbol{\phi}}^{\text {avail}}\right)$, as shown in (20). The reactive power (absorbing or injecting) is constrained by the power factor limit $\overline{\boldsymbol{P F}}_{\boldsymbol{g}}$, as shown in (21). Although the power factor is not explicitly modelled as a variable; it can be recovered using the relationship $P F_{g, \phi}=\tan ^{-1}\left(q_{g, \phi} / p_{g, \phi}\right)$.

$$
\begin{gathered}
\mathbf{0} \leq p_{g, \phi} \leq \boldsymbol{p}_{\boldsymbol{g}, \boldsymbol{\phi}}^{\text {avail }} \leq \overline{\boldsymbol{p}}_{\boldsymbol{g}, \boldsymbol{\phi}}, \forall g \in G, \phi \in \Phi \\
\left|q_{g, \phi}\right| \leq p_{g, \phi} \tan \left(\cos ^{-1} \overline{\boldsymbol{P F}}_{\boldsymbol{g}}\right), \forall g \in G, \phi \in \Phi
\end{gathered}
$$

Limits on the ramp rate is constrained by (22), where $\boldsymbol{p}_{\boldsymbol{g}, \boldsymbol{\phi}}^{\mathbf{0}}$ is the active power being injected and $\Delta \overline{\boldsymbol{p}}_{g, \phi}$ is the maximum change allowed (considering local regulations, connection agreements and the full ramping capability of the DG plant).

$$
\left|p_{g, \phi}-\boldsymbol{p}_{g, \phi}^{\mathbf{0}}\right| \leq \boldsymbol{\Delta} \overline{\boldsymbol{p}}_{\boldsymbol{g}, \boldsymbol{\phi}}, \forall g \in G, \phi \in \Phi
$$

Lastly, the control effort of DG plants is given by (23).

$$
u_{g}=\sum_{\phi \in \Phi}\left|q_{g, \phi}\right|, \forall g \in G, \phi \in \Phi
$$

The set of feasible tap positions of the OLTC is represented by $\Pi$ (indexed by $\pi$ ) and the tap ratio responding to each physical tap position is denoted by $\boldsymbol{\tau}_{\boldsymbol{t}, \boldsymbol{\pi}}$. A binary variable $k_{t, \pi} \in$ $[0,1]$ is introduced to determine whether tap $\pi$ is active.

Equation (24) ensures that only one tap can be active at any time and the voltage regulation capability can be modelled using (25) and (26). The Big M method is used in (25) and (26) where $\boldsymbol{M}$ is a relatively large constant; if p.u. values are used for voltages, $\boldsymbol{M} \geq 10$ is sufficient.

$$
\begin{gathered}
\sum_{\pi \in \Pi} k_{t, \pi}=1 \\
\mathrm{H}_{t} \geq 3\left(\boldsymbol{\tau}_{t, \pi}\right)^{2}\left[\begin{array}{lll}
v_{t, A}^{s e c} & v_{t, B}^{s e c} & v_{t, C}^{s e c}
\end{array}\right]^{t r}-\left(1-k_{t, \pi}\right) \boldsymbol{M} \\
\mathrm{H}_{t} \leq 3\left(\boldsymbol{\tau}_{t, \pi}\right)^{2}\left[\begin{array}{lll}
v_{t, A}^{s e c} & v_{t, B}^{\text {sec }} & v_{t, C}^{s e c}
\end{array}\right]^{t r}+\left(1-k_{t, \pi}\right) \boldsymbol{M} \\
\forall t \in T, \forall \pi \in \Pi
\end{gathered}
$$

The maximum number of tap changes allowed for each transformer $t$ (from its last tap position $\boldsymbol{\tau}_{\boldsymbol{t}}^{\mathbf{0}}$ ) can be limited by reducing the feasible values of $\pi$ in $\Pi$. This also helps to re- 
duce the number of integer variables $k_{t, \pi}$. The number of actual tap changes (i.e., the control effort $u_{t}$ ) can be calculated by finding the difference between the indices of the previous tap position $\boldsymbol{\pi}_{\boldsymbol{t}}^{\mathbf{0}}$ and new tap position $\pi_{t}^{*}$, as shown in (27).

$$
u_{t}=\left|\pi_{t}^{*}-\boldsymbol{\pi}_{\boldsymbol{t}}^{0}\right|, \forall t \in T
$$

\section{Solution Process}

The two-iteration approach (first discussed in [21]) is used to solve the proposed OPF problem in every control cycle. For the first iteration (first OPF run), the power flow results that are used to check for constraint violations (shown in Fig. 1) are also used as the estimated terms (i.e., the bolded terms with the $\sim$ accent). For the second iteration (second OPF run), the values of the estimated terms are replaced with the results from the first iteration. Finally, the results from the second iteration are used to extract the corresponding setpoints for controllable devices (e.g., tap positions for OLTCs).

The purpose of the second iteration is to improve the accuracy of the estimated terms, and thus the accuracy of the linearised OPF formulation. However, it is also possible to stop after the first iteration and extract the setpoints. Nonetheless, as demonstrated by our prior work in [22], the two-iteration approach offers accuracy improvements. This is because the linearisation point in the first iteration is based on recent measurements while the linearisation point in the second iteration considers the changes in network state due to the new setpoints (from the first iteration).

\section{CASE STUdy}

The performance of the proposed control scheme (which includes the adaptations discussed in Section II. is assessed using the generic UK distribution network EHV1. The analysis is carried out for a period of 24-hours using a realistic cosimulation platform and 1s time-step. Network simulation is carried out in OpenDSS [26] and the OPF problem is solved in AIMMS [27] using CPLEX 12.8. A Python [28] script is used to facilitate data exchanges between OpenDSS (i.e., the network) and AIMMS (i.e., the decision-making engine).

It is worth highlighting that this co-simulation environment allows the setpoints (calculated by the OPF) to be implemented in a network simulator (a proxy of a real distribution network), and thus realistically assess its adequacy. Furthermore, since the accuracy of the proposed linearizations has already been demonstrated in the authors' prior work [22], further discussions are omitted. Therefore, the assessment in this case study will focus on voltage compliance, congestion management, energy production, and the volume of control actions.

\section{A. EHVI Network}

The EHV1 network, a UK-style rural distribution network, is selected for this case study. A simplified diagram is shown in Fig. 5 and its data is available from [29]. It is designed to receive bulk power from the Grid Supply Point (GSP, also the slack bus) at $132 \mathrm{kV}$, distributing it through $33 \mathrm{kV}$ lines and, finally, arriving at $33 / 11 \mathrm{kV}$ primary substations. The aggregated peak demand (at $11 \mathrm{kV}$ ) is approximately $38 \mathrm{MW}$. All transformers, $132 / 33 \mathrm{kV}, 33 / 11 \mathrm{kV}$ and the voltage regulator

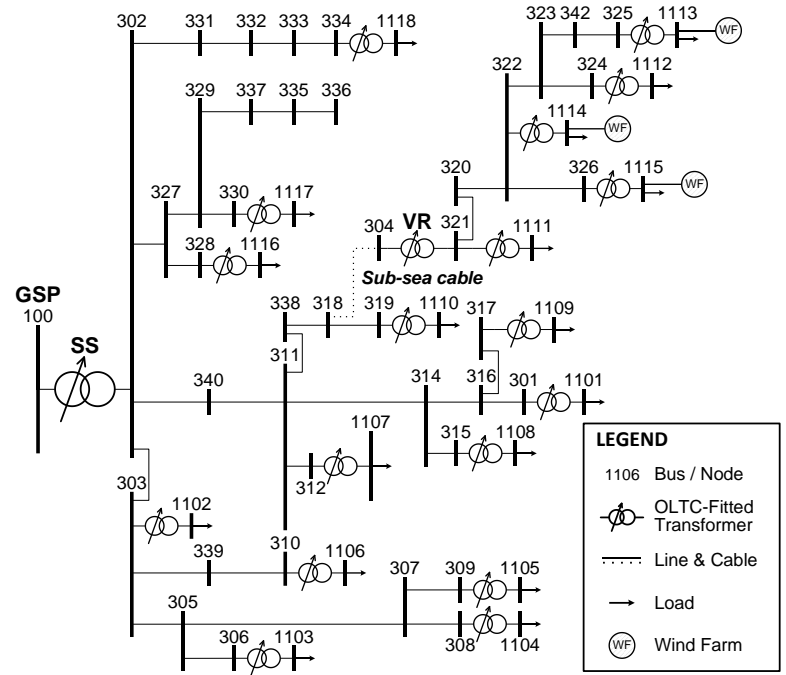

Fig. 5. EHV1 network diagram

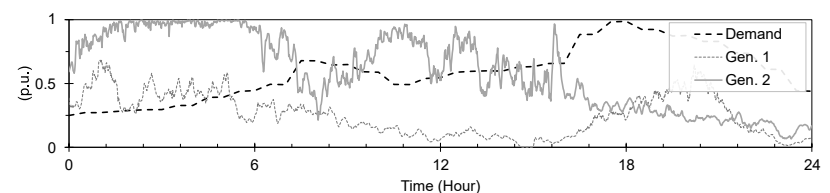

Fig. 6. Normalized demand and generation profiles

(VR), are fitted with OLTCs. Each OLTC has 20 taps, with $\boldsymbol{\tau}_{t, \pi} \in[0.85,1.05]$ in steps of 0.01 . There is also a delay of $3 \mathrm{~s}$ per tap change to account for the mechanical movements of contacts. The statutory steady-state voltage limits are $\pm 6 \%$ from the nominal for 11 and $33 \mathrm{kV}$ buses.

It is assumed that buses $1105,1106,1108,1113,1114$ and 1115 have been chosen as sites for wind farm connections. The investigated installed capacities are 8, 12, 4.5, 1.5, 7 and $12 \mathrm{MW}$, respectively, based on a previous planning study (albeit with hourly control actions) in the same context [9]. All wind farms are assumed to have reactive power capabilities of up to 0.95 power factor (inductive/capacitive).

Real wind (minute resolution) and demand (hourly) profiles are used, as shown in Fig. 6. Generation profile 1 is used for the first three wind farms and profile 2 is used for the latter three. To account for the geographical separations, the available profile used by one wind farm is shifted by 30 and 60 minutes to create two other profiles. The values of these demand and generation profiles are then linearly interpolated to achieve the 1s resolution used in this analysis. Lastly, for simplicity, generation and demand are allocated equally among the three phases of the corresponding $11 \mathrm{kV}$ buses.

\section{B. Modelling Considerations}

Instead of the statutory voltage limits, more conservative values ( $\pm 5.5 \%$ from the nominal) are used in (2) to account for numerical error due to the linear approximations. Furthermore, it is assumed desirable to keep voltages at the busbars after the primary substations above the nominal to compensate for voltage drops in the corresponding $11 \mathrm{kV}$ feeders (not explicitly modelled). Therefore, the lower voltage limit in (2) for buses 1101 to 1118 is then increased to $+0.5 \%$ from the nominal.

The sampling interval is set to $1 \mathrm{~s}$ (to match the simulation time-step). The length of the control cycles $\left(T_{C C}\right)$, the compu- 
tation time $\left(\Delta T_{\mathrm{OPF}}\right)$ and the communication delay $\left(\Delta T_{\mathrm{COM}}\right)$ are set to 120,5 and $5 \mathrm{~s}$, respectively. The desired execution time from each control action is set to $10 \mathrm{~s}$.

For wind farms, the transition to a new setpoint (both the active power and power factor) is executed linearly over $10 \mathrm{~s}$, in line with characteristics of wind turbine controllers [20]. The active power setpoint is implemented as an export limit that the wind farm operator must adhere to during each control cycle. The ramp rate restrictions (per connection agreement) is assumed to be $10 \%$ of the installed capacity per minute. The required ramping capability (from the wind farm) to achieve the desired response (dashed line in Fig. 3) is also assumed; typical inverter-interfaced wind farms have significantly higher ramping capability than the adopted connection agreement [30]. Hence, $\boldsymbol{\Delta} \overline{\boldsymbol{p}}_{\boldsymbol{g}, \boldsymbol{\phi}}=0.1 \times \overline{\boldsymbol{p}}_{\boldsymbol{g}, \boldsymbol{\phi}}$ is used in (22). For OLTCs under direct control, a limit of 3 taps per control cycle is imposed to match the execution time of DG Plants (i.e., $\Delta T_{\text {OLTC }} \approx \Delta T_{D G}$ ) and prevent large step changes in voltages by limiting the feasible values of $\Pi_{t}$ each time the OPF is solved.

The optimization engine uses the average SCADA readings from the last minute as the level of demand $\left(\boldsymbol{P}_{\boldsymbol{b}}\right.$ and $\left.\boldsymbol{Q}_{\boldsymbol{b}}\right)$ and wind resource availability ( $\boldsymbol{p}_{n}^{\text {avail }}$ ), i.e. persistent forecast.

The scaling factors used in the objective function are $\boldsymbol{c}_{\mathbf{1}}=$ $1 /\left(\sum_{g} \sum_{\phi} \boldsymbol{p}_{g, \phi}^{\text {avai }}\right), \quad \boldsymbol{c}_{2}=1 /\left(10 \sum_{t} 3\right) \quad$ and $\quad \boldsymbol{c}_{\mathbf{3}}=1 /$ $\left(100 \sum_{g} \sum_{\phi} \overline{\boldsymbol{p}}_{\boldsymbol{g}, \boldsymbol{\phi}} \tan \left(\cos ^{-1} \overline{\boldsymbol{P F}}_{\boldsymbol{g}, \boldsymbol{\phi}}\right)\right)$, which gives each term the range of $[0,1],[0,0.1]$ and $[0,0.01]$, respectively; i.e., it prioritizes the energy production (over control actions) and reactive power is preferred (over changing OLTCs' taps).

\section{Cases Investigated}

Two cases are considered to demonstrate the need of considering operating characteristics of controllable devices and the benefits of directly controlling OLTCs' taps. Case 1 adopts the conventional OLTC controller and corresponds to the typical setup in existing distribution networks. Consequently, since the proposed adaptations are not considered when implementing OPF-based setpoints, issues are expected in Case 1. For Case 1, the modelling of tap positions in (24)(27) is removed and the settings for each conventional OLTC controllers are shown in Table I. In contrast, the proposed adaptations are adopted in Case 2, and thus improvements are expected.

At the start of the simulation for both cases, the export limit and power factor of each DG plant are set to 5\% of the installed capacity and unity, respectively. Similarly, the initial tap ratio is set to 1.00 for all OLTCs.

\section{Results}

The performance of each case is discussed below, focusing on critical areas of the network, i.e., near the vicinity of the DG plants. Selected metrics are presented in Table II.

\section{1) Voltage Management}

Being a UK network, the BS EN50160 is used to assess voltage compliance, which states that $95 \%$ of the 10 -min RMS values must be within $[0.94,1.06]$ p.u. For simplicity, the assessment is done daily instead of weekly.
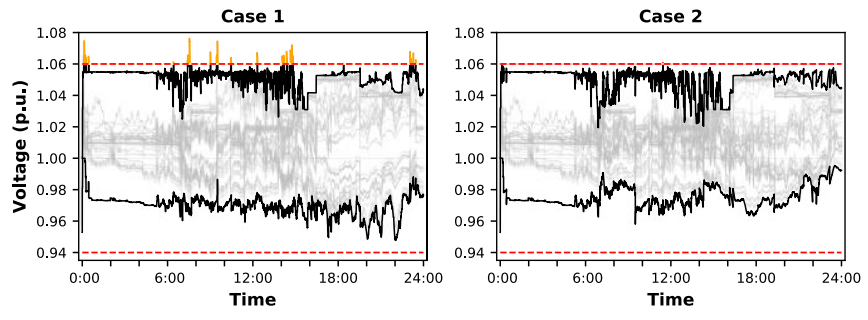

Fig. 7. Time-series voltage profiles
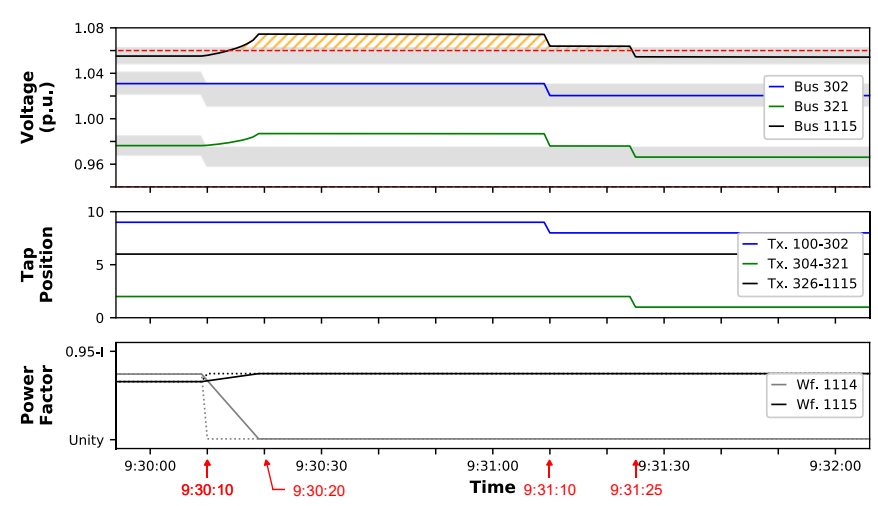

Fig. 8. Uncoordinated execution of setpoints (Case 1)

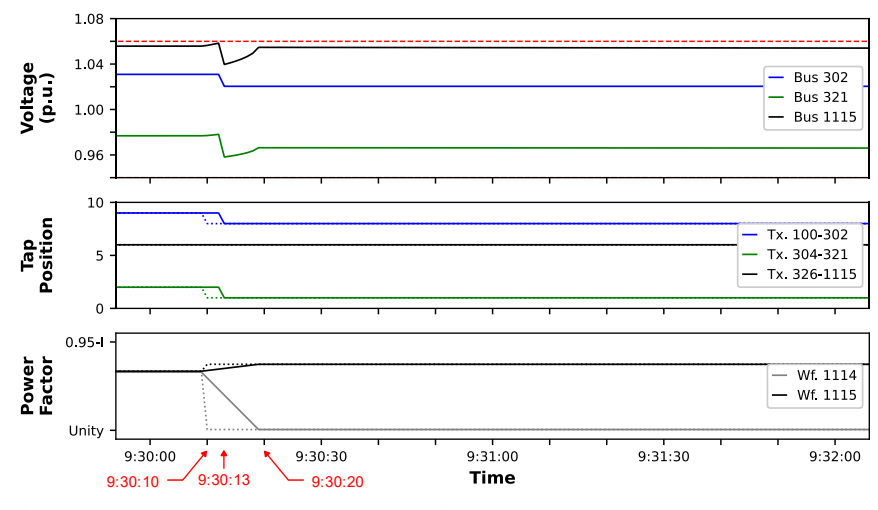

Fig. 9. Coordinated execution of setpoints (Case 2)

TABLE I

SETTINGS FOR CONVENTIONAL OLTC CONTROLLERS

\begin{tabular}{c|c|c|c}
\hline Transformer & Target & Delay & Deadband \\
\hline $132 \mathrm{kV} / 33 \mathrm{kV}(\mathrm{SS})$ & Variable & $60 \mathrm{~s}$ & $2.00 \%$ \\
\hdashline VR & Variable & $75 \mathrm{~s}$ & $1.75 \%$ \\
\hdashline $33 \mathrm{kV} / 11 \mathrm{kV}$ & Variable & $90 \mathrm{~s}$ & $1.50 \%$ \\
\hline
\end{tabular}

TABLE II

NETWORK-LeVEl PeRformance

\begin{tabular}{l|c|c}
\hline & Case 1 & Case 2 \\
\hline Maximum voltage (p.u.) & 1.075 & 1.06 \\
\hline Active power generated (MWh) & 408.6 & 408.6 \\
\hline Number of tap changes & 105 & 71 \\
\hline Reactive power absorbed (Mvarh) & 39.5 & 36.2 \\
\hline
\end{tabular}

When only considering the 10-min RMS values, voltage compliance is achieved $100 \%$ of the time for all the buses in both cases (results omitted). However, potential voltage issues become more apparent at $1 \mathrm{~s}$ resolution, as shown by the timeseries plots in Fig. 7. Here, the voltage profiles (at 1s granularity) of each bus is shown in grey, the envelope that encompasses all voltage profiles are shown in black, and the statutory limits are shown in red. Additionally, voltages above the statutory limit are emphasized in orange. From Fig. 7, frequent 
voltage spikes above the statutory limit (up to 1.075 p.u. as shown in Table II) are seen in Case 1. In reality, these excursions above the statutory limit may damage voltage sensitive equipment and interfere with other network devices with a voltage dependent response (e.g., having a Volt-Var curve). The severity of this will also depend on how restrictive the local standard is with respect to instantaneous voltages. On the other hand, the same issue is not observed in Case 2; voltages across the network are always kept within the statutory limits.

By examining the behaviors of OLTCs and DG plants in real-time, it is possible to demonstrate the importance of aligning their execution times. To illustrate this, Fig. 8 and Fig. 9 present the plots of a control cycle from 9:30:00 to 9:32:00 for Case 1 and Case 2, respectively. The upper plot shows the voltages at the secondary side of the $132 \mathrm{kV} / 33 \mathrm{kV}$ transformer 100-302 (in blue), the $33 \mathrm{kV} / 33 \mathrm{kV}$ transformer $304-321$ (in green) and $33 \mathrm{kV} / 11 \mathrm{kV}$ transformer $326-1115$ (in black). For Case 1, their corresponding voltage targets (considering the adopted deadbands using conventional OLTC controller) are also shown as shaded regions. The middle plot shows the actual tap positions. Lastly, the lower plot shows the real-time power factor (solid line) and the requested setpoint (dotted line) for the wind farm at bus 1115 (in black) and a nearby wind farm at bus 1114 (in grey).

Fig. 8 highlights how the uncoordinated execution of setpoints defined for this control cycle results in voltage spikes lasing over a minute. Since the transition to the new power factor is initiated immediately at 9:30:10 (after the 10s delay for $\Delta T_{\mathrm{OPF}}+\Delta T_{\mathrm{COM}}$ ), a sharp rise in bus voltage is observed from 9:30:10 to 9:30:20 due to a noticeably less inductive power factor being requested from wind farm 1114. Although lower voltage targets are also requested from the OLTCs of Tx. 100-302 and Tx. 304-321 to compensate for the rise in voltage, due to their delay (as per Table I), the responses did not occur for a further minute $\left(\Delta T_{\text {OLTC }}>60 \mathrm{~s}\right)$. As a result, voltage at bus 1115 spiked to over 1.075 p.u. (emphasized by the region in orange) until the responses from both Tx. 100302 and Tx. 304-321 are triggered (as shown in Fig. 8 starting from 9:31:10). Eventually, after both requested voltage targets are reached around 9:31:25, voltage at bus 1115 finally returned within the statutory limits.

In contrast, Case 2 recorded much better voltage profiles, as shown in Fig. 9. While the execution of setpoints also starts at 9:30:10 (same as Case 1) and the power factor has the same behavior (i.e., $\Delta \mathrm{T}_{D G}^{P F}$ is still at $10 \mathrm{~s}$ ), the over 1-min delay of the OLTCs is eliminated by controlling their taps directly. Given that the OPF requested one tap change from both Tx. 100-302 and Tx. 304-321, the new tap position is achieved by 9:30:13 (3s per tap, i.e., $\Delta T_{O L T C}=3 s$ ). Therefore, by enforcing similar execution times $\left(\Delta T_{O L T C} \approx \Delta \mathrm{T}_{D G}^{P F}\right)$, Case 2 successfully avoids the undesirable voltage spikes; the less inductive power factor is compensated for by the simultaneous decrease in voltage due to the OLTC, as originally intended.

\section{2) Congestion Management}

The utilization (as a percentage of the ampacity) of all lines and transformers is shown as grey lines in Fig. 10, along with the network-wide maximum (black line). Since OPF inherently considers the power flows within the network, each case manages to enforce appropriate export limits (based on the demand of the network) and, thus, ensure that the thermal constraint defined by $\boldsymbol{I}_{l}^{+}$in (8) is not violated throughout the day.

\section{3) Energy Production}

As expected, since the same primary objective is the same for both cases, the total energy produced from the wind farms (shown in Table II) has negligible differences; both are at 408.6 MWh for the simulated period.

\section{4) Volume of Control Actions}

As shown in Table II, the overall control effort of Case 1 is noticeably higher than Case 2. In summary, Case 2 achieved a significant reduction of $33 \%$ in tap changes (from 105 to 71 ) and a moderate reduction of $8.5 \%$ in reactive power absorption (from 39.5 Mvarh to 36.2 Mvarh).

There are two main contributing factors for the extra tap changes in Case 1: the relatively small deadband of conventional OLTC controllers and voltage drifts from an OLTC closer to the head of a feeder affecting downstream OLTCs. Due to these factors, conventional OLTCs controllers can be very ineffective when dealing with variable renewable resources. To this end, the differences in both cases are further examined by analyzing the time-series plots.

The generation and demand levels at bus 1115 are shown in Fig. 11. The behavior of each control scheme, in terms of the recorded control actions, are shown in Fig. 12 and Fig. 13, for Case 1 and Case 2, respectively. The voltage profiles (solid lines) at bus 321 (after Tx. 304-321) and 1115 (after Tx. 3261115) are shown in the upper and middle plots, respectively. For Case 1, the voltage targets of the corresponding transformers (shaded area) and the exact middle point of the deadband (dotted line) are also shown. The tap position of Tx. 3261115 is shown in the lower plot.

Due to a steadily decrease of wind resources for the wind farm at bus 1115 (Fig. 11), the control scheme in Case 1 attempted to reduce the number of tap changes by exploiting the available voltage headroom. Theoretically, this can be achieved by setting a voltage target that tracks the busbar voltage at the secondary side of each OLTC-fitted transformer. However, in practice, voltage variations (from the requested value) due to the deadband of up-stream OLTCs (e.g., Tx. 304-321) will make this approach less effective for any downstream OLTCs (e.g., Tx. 325-1115). This can be seen by examining the voltages at bus 321 in Fig. 12; albeit still within the deadband, the actual voltage is consistently lower than requested (below the dotted line). This, in turn, makes voltages downstream, such as at bus 1115 , to be lower from what the optimization engine expects (i.e., middle of the deadband). Therefore, although the voltage target is continuously adjusted for Tx. 326-1115 (as shown by the step changes of the shaded area in Fig. 12), it is rather ineffective. The relatively small deadband of Tx. 326-1115 further exacerbates the issue. Overall, three tap changes are recorded (between 6:45 and 7:05) despite an attempt to minimize control actions. 
On the other hand, in Case 2 (Fig. 13), the exact tap position is requested to eliminate the issues associated with the OLTCs' deadbands (as seen in previous cases). Consequently, the available voltage headroom is effectively exploited to eliminate unnecessary tap changes for Tx. 326-1115; during the same period (from 6:45 to 7:05), no tap changes are needed. As presented in Table II, the overall improvement is also quite substantial, a $33 \%$ reduction compared with Case 1.

\section{E. Remarks on Solution Speed}

The barrier method is used to solve the LP relaxation at the root node (due to the sparsity of the matrix passed on to the solver) whereas the simplex method is used during the branchand-cut stage (to take advantage of advanced basis).

Overall, the OPF problem (formulated as a MILP) is solved to optimal with an average computation time of $0.46 \mathrm{~s}$ (for the adopted two iterations) on a typical PC with an Intel i5 processor. The maximum recorded is $1.2 \mathrm{~s}$.

\section{DISCUSSION}

The availability of network modelling data and communication infrastructure is assumed in this work. Furthermore, state-estimation may be needed in the absence of full network observability. However, this is beyond the scope of this work.

The use of control cycles lasting a few minutes has been adopted as an effective way to mitigate violations of network constraints $[6,18]$ thanks to limited variations of minute-tominute outputs from typical renewable DG plants [6, 31]. Nonetheless, variations are still expected within each control cycle which, in turn, can cause constraint violations. To this end, the probability and the extent of these variations as well as the need for additional modelling to cater for the uncertainties should be assessed (e.g., based on historical data). At the same time, this also entails the need for more granular data (e.g., time resolution of seconds) to realistically capture this phenomenon as the averaging effect of lower resolutions data (e.g., minutes) will mask such spikes or fluctuations. Lastly, apart from using control cycles, alternative approaches to trigger the decision-making process can be adopted, such as the problem persistency mechanism used in [32].

This work has focused on two common avenues of tackling voltage issues in distribution networks, i.e., OLTCs and reactive power from DG plants. While beyond the scope of this work, it is worth highlighting that other sources of flexibility also exist (or starting to emerge) such as demand response, battery storage and network-interacting microgrids. To this end, this work serves as a starting point to further investigate the necessary practical considerations when other sources of flexibility are to be considered by DNOs in the future, particularly when different types of technologies are being leveraged at the same time.

In this work, the wind farms are modelled as PQ sources, a common practice in distribution network studies and is typically sufficient for quasi-static time-series simulations that focuses on the control aspect in the second-scale [33]. If needed, more detailed modelling of the electromechanical characteristics (e.g., wind turbines or inverters) can be incorporated.
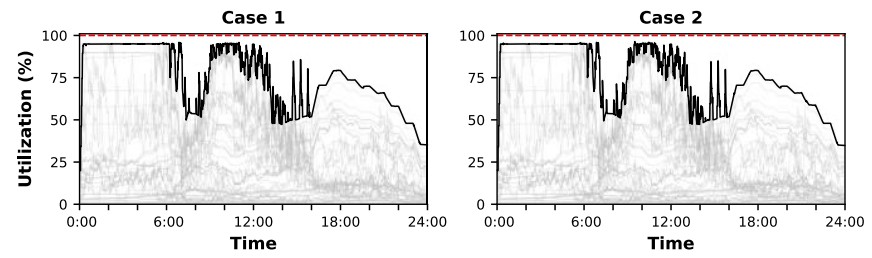

Fig. 10. Utilization of lines and transformers

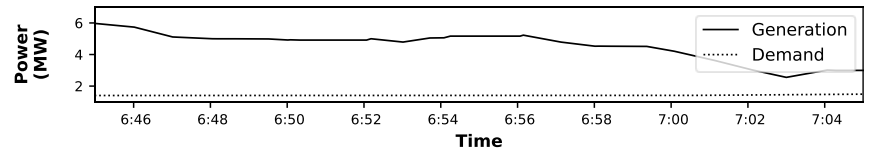

Fig. 11. Generation and demand at bus 1115
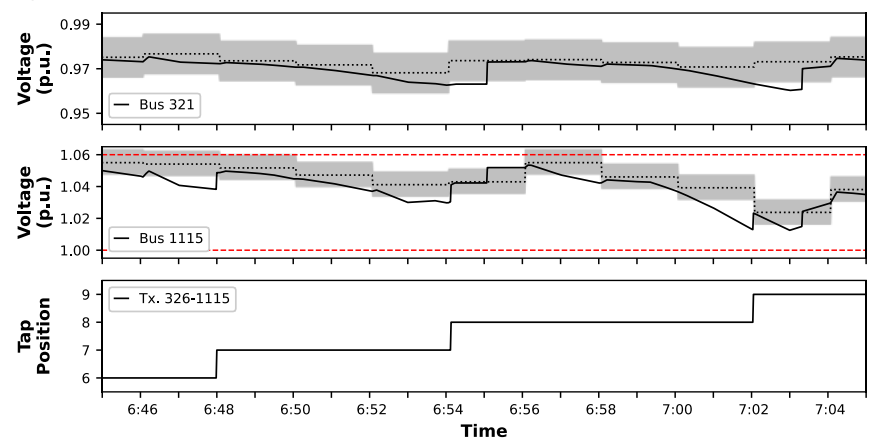

Fig. 12. OLTC behavior with conventional controller (Case 1)
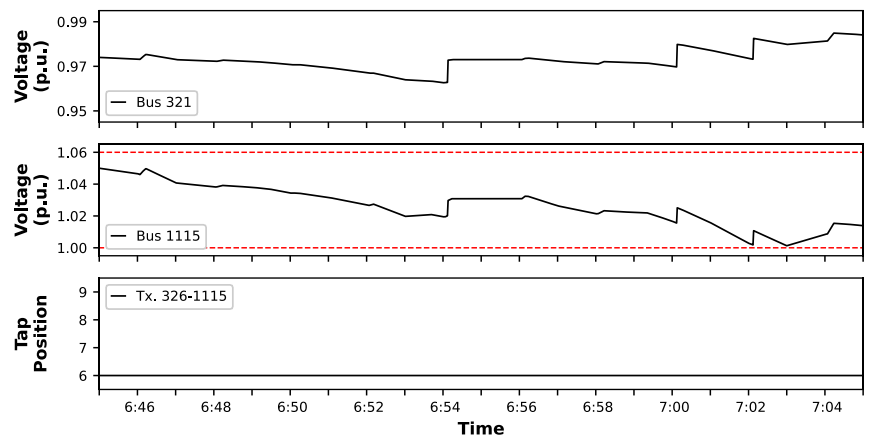

Fig. 13. OLTC behavior with direct tap control (Case 2)

Nonetheless, regardless of the modelling details of wind farms, the coordination issue investigated in this work (i.e., between wind farms and OLTCs) will still apply.

An extension of this work is to consider other emerging sources of flexibility in distribution networks, such as battery storage and real-time network reconfiguration. Since these sources of flexibility can exhibit different operating characteristics to the devices investigated in this work, the proposed methodology can help to ensure the OPF-based setpoints are adequately implemented in practice. It is also worth mentioning that, while an OPF formulated using the branch flow model has limitations when dealing with a meshed network (e.g., required when catering for network reconfiguration), an alternative formulation (e.g., as in [32]) can be used without affecting the validity of the proposed adaptations. This is because these adaptations focus on the behavior of devices, not the power flow equations.

The terms related to control actions in the objective function, i.e., in (1), mainly serve as penalty factors to prevent unnecessary control actions of OLTCs and DG reactive power 
while not affecting the objective of maximizing renewable energy harvesting. This is needed because there are often multiple sets of control actions to achieve the same volume of energy harvesting. Nonetheless, in terms of the adopted weighting factors, a sensitivity analysis would be prudent for each case study as inadequate values can lead to undesirable outcomes. Furthermore, there are alternative ways of formulating the objective function (such as based on costs). This also introduces opportunities to explore other approaches that consider the trade-offs among multiple objectives (such as a Pareto optimal approaches).

Finally, the analysis in the case study has focused on technical metrics only (e.g., kWh and volume of control actions). While a cost-benefit analysis is another important aspect, it is worth highlighting that the cost associated with curtailment and the technologies involved (e.g., direct control of OLTCs) will depend on how a DNO values them. This, in turn, depends on local prices, policies and regulation. Nonetheless, for a given scenario where accurate cost data is available, an economic assessment is also prudent to achieve a more complete assessment for that scenario.

\section{CONCLUSION}

Although considerable literature exists on AC Optimal Power Flow (in the context of active distribution networks), issues arising from how the calculated setpoints are actually implemented by controllable devices have not been adequately investigated. As demonstrated here, the uncoordinated control of on-load tap changers (OLTCs) and Distributed Generation (DG) plants to mitigate voltage issues may cause voltage spikes instead, due to a mismatch in their execution times. Furthermore, the deadbands of conventional OLTCs introduce voltage drifts around the requested voltage target and renders OPF-based setpoints less effective in attempting to minimize control actions.

To this end, this work has proposed a series of necessary adaptations within existing device controllers and the OPF formulation to ensure the centrally dispatched setpoints are adequately executed locally by controllable devices. From the device perspective, this requires understanding and modifying their operating characteristics (i.e., delay, ramp rate and deadband) to ensure better temporal coordination among devices. Particularly, the direct control of conventional devices and a new ramping behavior for inverter-interfaced devices are proposed. Furthermore, a linear, mixed-integer OPF formulation is adopted to handle discrete devices and improve scalability in large networks.

The effectiveness of the proposed changes is demonstrated using a realistic co-simulation platform and 1s time-resolution to capture the intricate interactions among controllable devices in the sub-minute scale. Results (using a UK test network with the objective of maximizing renewable energy production) have shown that, by catering for the diverse operating characteristics of controllable devices, the expected outcome from OPF-based setpoints can be achieved. These findings will allow Distribution Network Operators to better understand the different aspects that advanced, centralized approaches need to cater for when implemented in their networks.

\section{APPENDIX}

The linearized voltage drop equation in (3) is derived from the complex three-phase voltage drop equation in (28) [25].

$$
\vec{V}_{l_{x}, \phi}-\vec{V}_{l_{y}, \phi}=\sum_{\psi \in \Phi} \vec{Z}_{l, \phi, \psi} \vec{S}_{l_{x}, \psi}^{*} / \vec{V}_{l_{x}, \psi}^{*}
$$

By taking the magnitude of both sides of (28) and rearranging terms as necessary, the quadratic voltage drop equation, i.e., (29), and equivalent impedance, i.e., (30) and (31), can be obtained. Similar to (3), the auxiliary terms $\kappa_{1}^{\#}, \kappa_{2}^{\#}$ and $\kappa_{3}^{\#}$ are introduced for compactness and the superscript $\#$ is used to differentiate these auxiliary terms from the ones in (3).

$$
\begin{gathered}
v_{l_{x}, \phi}-v_{l_{y}, \phi}=2 \kappa_{1}^{\#}-\left[\left(\kappa_{1}^{\#}\right)^{2}+\left(\kappa_{2}^{\#}\right)^{2}\right] / v_{l_{x}, \phi} \\
\kappa_{1}^{\#}=\sum_{\psi \in \Phi}\left(R_{l, \phi, \psi}^{\#} P_{l_{x}, \psi}+X_{l, \phi, \psi}^{\#} Q_{l_{x}, \psi}\right) \\
\kappa_{2}^{\#}=\sum_{\psi \in \Phi}\left(X_{l, \phi, \psi}^{\#} P_{l_{x}, \psi}-R_{l, \phi, \psi}^{\#} Q_{l_{x}, \psi}\right) \\
R_{l, \phi, \psi}^{\#}=\left[\boldsymbol{R}_{l, \phi, \psi} \cos \left(\kappa_{3}^{\#}\right)-\boldsymbol{X}_{l, \phi, \psi} \sin \left(\kappa_{3}^{\#}\right)\right] V_{l_{x}, \phi} / V_{l_{x}, \psi} \\
X_{l, \phi, \psi}^{\#}=\left[\boldsymbol{R}_{l, \phi, \psi} \sin \left(\kappa_{3}^{\#}\right)+\boldsymbol{X}_{l, \phi, \psi} \cos \left(\kappa_{3}^{\#}\right)\right] V_{l_{x}, \phi} / V_{l_{x}, \psi} \\
\kappa_{3}^{\#}=\angle \vec{V}_{l_{x}, \psi}-\angle \vec{V}_{l_{x}, \phi}
\end{gathered}
$$

Using estimated values for $V_{l_{x}, \phi}, V_{l_{x}, \psi}, \angle \vec{V}_{l_{x}, \psi}$ and $\angle \vec{V}_{l_{x}, \phi}$ (e.g., the power flow results per Fig. 1), (30) and (31) become the constants as shown in (6) and (7), respectively. The final non-linear term in (29), i.e., $\left[\left(\kappa_{1}^{\#}\right)^{2}+\left(\kappa_{2}^{\#}\right)^{2}\right] / v_{l_{x}, \phi}$, can be linearized using estimated values for $\kappa_{1}^{\#}, \kappa_{2}^{\#}$ and $v_{l_{x}, \phi}$ to obtain the final form shown in (3). Since $\left[\left(\kappa_{1}^{\#}\right)^{2}+\left(\kappa_{2}^{\#}\right)^{2}\right] / v_{l_{x}, \phi}$ is related to the losses and is much smaller in magnitude than the other terms, the overall impact on accuracy is insignificant.

The power flow expressions in (4) and (5) are derived from the losses equation in (32) [25]. By extracting the active and reactive components as well as applying the same technique of using estimated terms, (4) and (5) can be obtained.

$$
\vec{S}_{l_{x}, \phi}-\vec{S}_{l_{y}, \phi}=\sum_{\psi \in \Phi}\left(\vec{Z}_{l, \phi, \psi} \vec{S}_{l_{x}, \psi}^{*} / \vec{V}_{l_{x}, \psi}^{*}\right) \cdot \vec{S}_{l_{x}, \phi} / \vec{V}_{l_{x}, \phi}
$$

The power relationships of delta-wye transformers in (15) and (16) are derived from the phasor power balance equation; an example is shown in (33) for phase A [24]. The matrix form in (15) and (16) can be obtained by separating the real and imaginary components and the following approximations: $\left|\vec{V}_{t, A}^{\text {pri }}\right| \approx\left|\vec{V}_{t, C}^{\text {pri }}\right|$ and $\angle \vec{V}_{t, C}^{\text {pri }}-\angle \vec{V}_{t, A}^{\text {pri }} \approx 120^{\circ}$.

$$
\vec{S}_{t, A}^{\text {pri }}=\vec{S}_{t, A}^{s e c} \vec{V}_{t, A}^{\text {pri }} /\left(\vec{V}_{t, A}^{\text {pri }}-\vec{V}_{t, C}^{\text {pri }}\right)
$$

Finally, the linearised voltage relationships of delta-wye transformers in (17) is derived from the phasor relationships between the primary and secondary terminals; an example is shown in (34) for phase A [25]. For a balanced system, equation (17) without the second column vector in $H_{t}$ can be obtained by taking the magnitude of (34) and using the identity $\vec{V}_{t, A}^{\text {pri }}+\vec{V}_{t, B}^{\text {pri }}+\vec{V}_{t, C}^{\text {pri }}=0$. While this does not hold due to phase unbalances, an offset vector $\vec{V}_{t, O}^{\text {pri }}=\vec{V}_{t, A}^{\text {pri }}+\vec{V}_{t, B}^{\text {pri }}+\vec{V}_{t, C}^{\text {pri }}$ can be introduced to derive the full equation in (17). Particularly, the second column vector in $H_{t}$ is used to approximate the magnitude of $\vec{V}_{t, O}^{p r i}$, and thus increases the overall accuracy.

$$
\sqrt{3} \tau_{t} \vec{V}_{t, A}^{s e c}=\vec{V}_{t, A}^{p r i}-\vec{V}_{t, C}^{p r i}
$$




\section{REFERENCES}

[1] R. Currie, D. Macleman, G. McLorn, and R. Sims, "Operating the orkney smart grid: Practical experience," in Proc. 2011 21st International Conference on Electricity Distribution (CIRED).

[2] G. C. Kryonidis, E. O. Kontis, A. I. Chrysochos, C. S. Demoulias, and G. K. Papagiannis, "A Coordinated Droop Control Strategy for Overvoltage Mitigation in Active Distribution Networks," IEEE Trans. Smart Grid, vol. 9, no. 5, pp. 5260-5270, 2018.

[3] Y. Xu, Z. Y. Dong, R. Zhang, and D. J. Hill, "Multi-Timescale Coordinated Voltage/Var Control of High Renewable-Penetrated Distribution Systems," IEEE Trans. Power Syst., vol. 32, no. 6, pp. 4398-4408, 2017.

[4] S. Gill, I. Kockar, and G. W. Ault, "Dynamic Optimal Power Flow for Active Distribution Networks," IEEE Trans. Power Syst., vol. 29, no. 1, pp. 121-131, Sept. 2014.

[5] Y. J. Kim, J. L. Kirtley, and L. K. Norford, "Reactive Power Ancillary Service of Synchronous DGs in Coordination With Voltage Control Devices," IEEE Trans. on Smart Grid, vol. 8, no. 2, pp. 515-527, 2017.

[6] S. W. Alnaser and L. F. Ochoa, "Advanced Network Management Systems: A Risk-Based AC OPF Approach," IEEE Trans. Power Syst., vol. 30, no. 1, pp. 409-418, May 2015.

[7] T. Soares, R. J. Bessa, P. Pinson, and H. Morais, "Active Distribution Grid Management Based on Robust AC Optimal Power Flow," IEEE Trans. Smart Grid, vol. 9, no. 6, pp. 6229-6241, 2018.

[8] A. O'Connell, A. Soroudi, and A. Keane, "Distribution Network Operation Under Uncertainty Using Information Gap Decision Theory," IEEE Trans. Smart Grid, vol. 9, no. 3, pp. 1848-1858, 2018.

[9] L. F. Ochoa, C. J. Dent, and G. P. Harrison, "Distribution Network Capacity Assessment: Variable DG and Active Networks," IEEE Trans. Power Syst., vol. 25, no. 1, pp. 87-95, Nov. 2010.

[10] A. Kulmala, S. Repo, and P. Järventausta, "Coordinated Voltage Control in Distribution Networks Including Several Distributed Energy Resources," IEEE Trans. on Smart Grid, vol. 5, no. 4, pp. 2010-2020, 2014.

[11] S. N. Salih and P. Chen, "On Coordinated Control of OLTC and Reactive Power Compensation for Voltage Regulation in Distribution Systems With Wind Power," IEEE Transactions on Power Systems, vol. 31, no. 5, pp. 4026-4035, 2016.

[12] Implementation of active voltage and active power flow management with FPP Trial area. [Online]. Available: https://innovation.ukpowernetworks.co.uk/

[13] High-Level Design Specification of Advanced Automation Solution. [Online]. Available: https://innovation.ukpowernetworks.co.uk/

[14] A. R. Ahmadi, M. Gordon, M. White, A. Minton, S. Georgiopoulos, and D. Stamatiadis, "Enhanced Transmission and Distribution System Coordination and Control Utilising Distribution Network Capacity and Avoiding Conflicts of Service Offered to Transmission System Operator," 2019

[15] M. Farivar and S. H. Low, "Branch Flow Model: Relaxations and Convexification\&\#x2014;Part I," IEEE Transactions on Power Systems, vol. 28, no. 3, pp. 2554-2564, 2013.

[16] Y. Tang, K. Dvijotham, and S. Low, "Real-Time Optimal Power Flow," IEEE Trans. Smart Grid, vol. 8, no. 6, pp. 2963-2973, 2017.

[17] E. Dall'Anese and A. Simonetto, "Optimal Power Flow Pursuit," IEEE Trans. Smart Grid, vol. 9, no. 2, pp. 942-952, 2018.

[18] J. Robertson, G. Harrison, and R. Wallace, "Receding-horizon OPF for real-time management of distribution networks," IET Generation, Transmission \& Distribution, vol. 12, no. 9, pp. 2124-2131, 2018.

[19] A. Ballanti, L. N. Ochoa, K. Bailey, and S. Cox, "Unlocking New Sources of Flexibility: CLASS: The World's Largest Voltage-Led LoadManagement Project," IEEE Power and Energy Magazine, vol. 15, no. 3, pp. 52-63, 2017.

[20] K. Clark, N. W. Miller, and J. J. Sanchez-Gasca, "Modeling of GE Wind Turbine-Generators for Grid Studies," GE Energy, Schenectady, NY, Apr. 16, 2010.

[21] J. F. Franco, L. F. Ochoa, and R. Romero, "AC OPF for Smart Distribution Networks: An Efficient and Robust Quadratic Approach," IEEE Trans. Smart Grid, vol. 9, no. 5, pp. 4613-4623, 2018.

[22] L. Gutierrez-Lagos, M. Z. Liu, and L. F. Ochoa, "Implementable ThreePhase OPF Formulations for MV-LV Distribution Networks: MILP and MIQCP," in Proc. 2019 IEEE PES Innovative Smart Grid Technologies Conference - Latin America (ISGT Latin America).
[23] M. Baran and F. F. Wu, "Optimal sizing of capacitors placed on a radial distribution system," IEEE Transactions on Power Delivery, vol. 4, no. 1 , pp. 735-743, 1989.

[24] C. Tsai-Hsiang and C. Yung-Liang, "Integrated models of distribution transformers and their loads for three-phase power flow analyses," IEEE Trans. Power Del., vol. 11, no. 1, pp. 507-513, 1996.

[25] W. H. Kersting, Distribution System Modeling and Analysis, Fourth edition. ed: CRC Press, 2017.

[26] R. C. Dugan and T. E. McDermott, "An open source platform for collaborating on smart grid research," in Proc. 2011 IEEE Power and Energy Society General Meeting, pp. 1-7.

[27] AIMMS. [Online]. Available: https://www.aimms.com/

[28] Python. [Online]. Available: https://www.python.org

[29] United Kingdom Generic Distribution System. [Online]. Available: https://github.com/sedg/ukgds

[30] G. Abad, J. Lopez, M. Rodriguez, L. Marroyo, and G. Iwanski, Doubly fed induction machine: modeling and control for wind energy generation, vol. 85: John Wiley \& Sons, 2011.

[31] Y. Wan, "Analysis of Wind Power Ramping Behavior in ERCOT," National Renewable Energy Laboratory, Golden, CO, Mar., 2011.

[32] L. Gutierrez-Lagos and L. F. Ochoa, "OPF-Based CVR Operation in PVRich MV-LV Distribution Networks," IEEE Transactions on Power Systems, vol. 34, no. 4, pp. 2778-2789, 2019.

[33] IEEE Guide for Conducting Distribution Impact Studies for Distributed Resource Interconnection, IEEE P1547.7, 2013

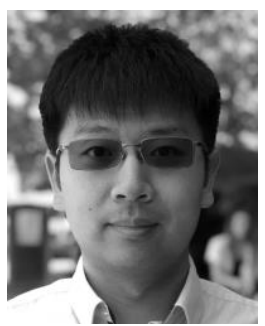

Michael Z. Liu (S'10) received his B.Eng. (Hons) degree in 2012 and Ph.D. degree in 2020, both from The University of Melbourne, Melbourne, VIC, Australia. He is currently a Postdoctoral Researcher with The University of Melbourne. His research interests include distribution network modelling, real-time orchestration of distributed energy resources, and three-phase optimal power flow formulations.

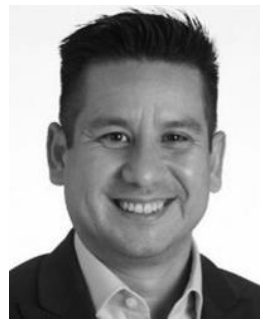

Luis F. Ochoa (S'01-M'07-SM'12) received the B.Eng. degree from the Universidad Nacional de Ingenier' 1 a, Lima, Peru, in 2000 and the M.Sc. and Ph.D. degrees from the Universidade Estadual Paulista, Ilha Solteira, Brazil, in 2003 and 2006, respectively. He is currently a Professor of Smart Grids and Power Systems with the University of Melbourne, Melbourne, VIC, Australia, and a part-time Professor of Smart Grids with the University of Manchester, Manchester, U.K. His research interests include network integration and control of distributed energy resources and future low-carbon distribution networks.

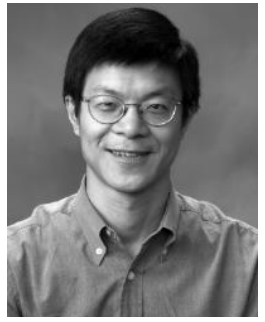

Steven H. Low (F'08) is the Gilloon Professor of Computing and Mathematical Sciences and Electrical Engineering with the California Institute of Technology, Pasadena, CA, USA, and Honorary Professor of Melbourne University, VIC, Australia. He is a Fellow of IEEE and ACM. His research on networking has been accelerating more than $1 \mathrm{~TB}$ of Internet traffic every second since 2014. His research on smart grid is providing large-scale electric 1010 vehicle charging to workplaces, from K-12 and universities to Fortune Global 500 companies. He received his BS from Cornell University, Ithaca, NY and PhD from UC Berkeley, CA, both in EE. 